\title{
Progressing to the new normal: Teaching in radiology during COVID-19 at a major institution in Mexico
}

\author{
Franklin O. Ordonez-Rivas ${ }^{1}$, Claudio Casas-Murillo ${ }^{1}$, Elliott J. Winford ${ }^{2}$, Natalia Villarreal-Del Bosque ${ }^{1}$, \\ Matías Salinas-Chapa ${ }^{1}$, Bhavya Rehani ${ }^{3}$, and Guillermo Elizondo-Riojas ${ }^{1 *}$ \\ ${ }^{1}$ Department of Radiology, Universidad Autónoma de Nuevo León, Hospital Universitario "Dr. José Eleuterio González", Monterrey, Nuevo León, \\ Mexico; ${ }^{2}$ School of Medicine, Meharry Medical College, Nashville, Tennessee, United States of America; ${ }^{3}$ Department of Radiology and Biomedical \\ Imaging, University of California, San Francisco, San Francisco, California, United States of America
}

\begin{abstract}
Background: SARS-CoV-2 poses challenges to medical education. Radiology is remarkably poised for such burdens, given its considerable dependence on various technologies. Objective: The objective of the study was to investigate infrastructural and procedural changes in a radiology department (RD) in response to the pandemic and the academic impact these changes generated. Methods: The RD of the UANL sustained significant alterations to maintain its residency program's integrity. Explored modifications include: Implementing remote learning opportunities, real-time software and technologies, and virtually administered competency evaluations. Results: Despite departmental adaptations, the RD preserved the overall quality of resident education with an increased specialty in June and July of $65.2 \%$. The total number of academic activities more than doubled compared to June and July of 2019. Remote technologies allow for the continuance of medical training and maintained interactions with peers and attending physicians. Conclusion: Radiology training must continue amidst the pandemic. For this to occur, traditional radiology training must change. Interventions must preserve the educational integrity of the residency programs while minimizing risk to trainees. While these changes were necessary to combat viral spread, their proven utility may prompt sustained medical training integration.
\end{abstract}

Key words: SARS CoV-2. Radiology education.Post-pandemic education. Medical education.New normality in medical education

\section{Introduction}

SARS-CoV-2 (coronavirus) has severe societal implications. The viral spread has occurred at an unprecedented rate, with nearly every country reporting confirmed cases within a matter of months. On February 28, 2020, Mexico confirmed its first known case of COVID-19. Despite extensive country-wide measures, Mexico has yet to achieve an identifiable plateau concerning daily new cases or deaths. Given an ever-rising patient burden, the country's health-care system has all but reached maximum capacity. Concerns for provider safety have only further complicated the delivery of health-care services, necessitating innovative measures.

The novel coronavirus also poses significant challenges to medical education. Residency programs globally have struggled to meet heightened provider demand while not subjecting trainees to unnecessary risk. The pandemic's novelty has led to a non-uniform global response in this regard, with many program directors now being forced to cope in ways that potentially

\section{Correspondence:}

*Guillermo Elizondo-Riojas

E-mail: guillermo.elizondorj@uanl.edu.mx
Date of reception: 03-07-2021

Date of acceptance: 01-10-2021 DOI: 10.24875/RMU.21000029
Available online: 01-12-2021 Medicina Universitaria. 2019;21(1):128-132 www.medicinauniversitaria.org license (http://creativecommons.org/licenses/by-nc-nd/4.0/). 
compromise the academic rigor of their respective programs.

Radiology is perhaps, of all the medical specialties, best suited to adapt to the COVID-19 era, given its already extensive integration with available technologies. However, literature that elucidates how best to utilize such resources remains scarce. The radiology department (RD) of the Autonomous University of Nuevo León worked swiftly during the pandemic to promote changes in their radiology residency program's infrastructure. Although only implemented at a single hospital, the reported interventions are likely applicable to other institutions.

Health4theWorld and the RD at the UANL collaborated during these challenging times, culminating in creating the Health4theWorld Radiology Chapter at UANL. The COVID-19 educational initiatives adopted at the UANL were discussed in a COVID-19 preparedness series of webinars so that trainees worldwide could benefit from the innovative educational models adopted at the UANL.

\section{Materials and methods}

A period of preparation between March and April was required to develop strategies and protocols needed to keep the residency program active. Specifically, the department aimed to respond to the pandemic in the following ways:

Guiding Principles:

1. Minimize interruptions in trainee education as much as can feasibly be done

2. Provide working environments for residents that do not compromise safety

3. Devise interventions that do not hinder critical interactions between trainees and faculty

4. Utilize other educational and communicative modalities to promote maintenance of departmental functionality.

\section{Interventions}

Drastic measures were necessary to reduce hospital presence. The department implemented a split schedule whereby half of all radiology trainees were to report to the hospital while the others were instructed to remain home. The department scheduled the groups to switch locations every 2 weeks, allowing an equitable distribution of in-person training.

For home-based trainees, the department established workstations within their respective locations. Residents were required to engage in clinical duties for a minimum of $8 \mathrm{~h} /$ day, with mandatory monthly progress reports, including reviews by professors of the radiology service, interesting case discussions, etc. (Fig. 1A). A hospital enabled computer, a portable tablet (Fig. 1B), a webcam, software such as YouTube Live (YouTube, LLC, San Bruno, CA. 2013) and Microsoft Teams (Microsoft Corporation, Redmond, WA. 2017), a media streaming program, and a collaborative platform were all included. In addition to image interpretation, clinical responsibilities included compulsory morning sessions with classes scheduled for the whole year taken from the curriculum of European and American Radiology Society, Grand Rounds every day, Rad Primers assignments, and Rad Primers reading time, accumulating at least $20 \mathrm{~h}$ of reading time per day, and tumor boards with other hospital services.

As the number of patients received by the hospital has decreased significantly since the pandemic's onset, it was impossible to equitably compare the number of studies performed before and during COVID-19. Howev$\mathrm{er}$, radiology residents were required to interpret all daily radiological studies assigned at each workstation.

\section{Results}

Following the establishment of a new online format for academic activities, the RD observed a $30.7 \%$ increase in the total number of activities held from March to July (Table 1). Independent activities such as intradepartmental sessions, tumor boards, Grand Rounds, and webinars also observed improvements. While tumor boards notably doubled in frequency from 2019 (19 vs. 38 ), the number of Grand Rounds (0 vs. 43) and webinars ( 6 vs. 41 ) were the most pronounced changes.

Activities held by the department for specific months also showed trends. The year 2019 had no webinars from March to June (Fig. 2). For the year 2020, April was the only month without a webinar, with all other months showing increases compared to 2019. Besides these, the RD held intradepartmental sessions with increasing frequency during each month of the pandemic, contrasting the variable pattern observed in the prior calendar year (Fig. 3).

The early stages of the pandemic experienced a decrease in the frequency of academic activity. A 26.2\% reduction was noted in total educational activities for March 2020 (30) compared to March 2019 (42). This trend was also apparent for April, sustaining a 33.3\% reduction. 
Table 1. Total amount of academic activities made between March-July in 2019 and 2020

\begin{tabular}{|c|c|c|c|c|c|c|}
\hline Year & Intradepartamental Sessions & Tumor Boards & Interdepartamental Sessions & Grand Rounds & Webinars & Total \\
\hline 2019 & 90 & 38 & 71 & 0 & 6 & 205 \\
\hline 2020 & 101 & 19 & 64 & 43 & 41 & 268 \\
\hline
\end{tabular}

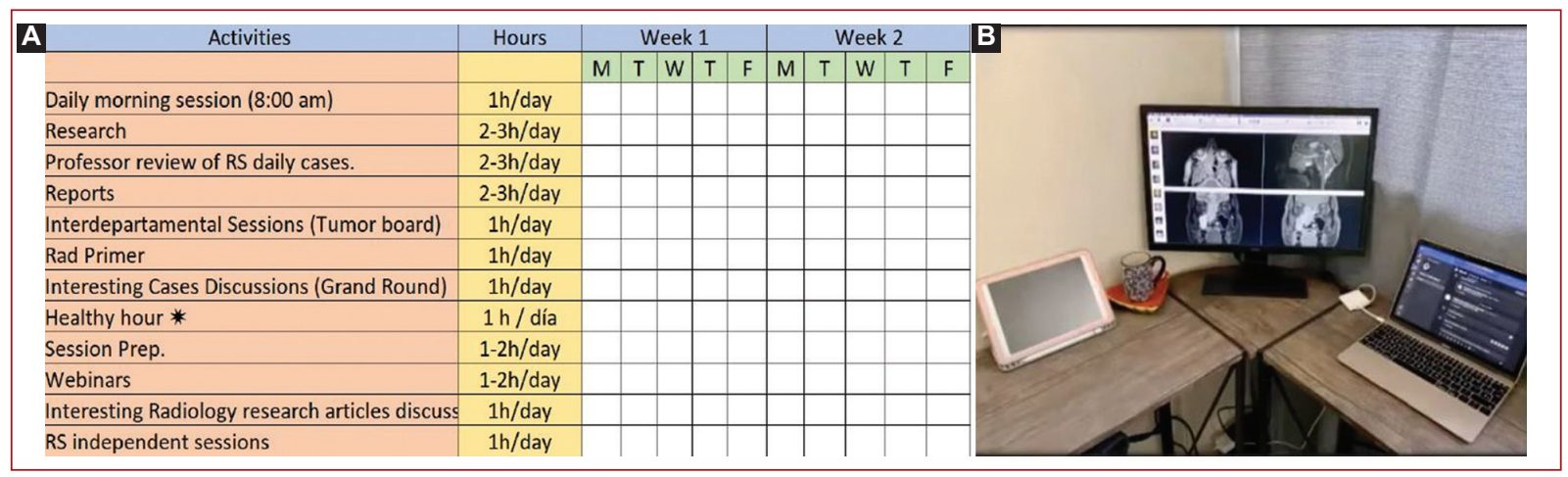

Figure 1. A: trainees were required to engage in activities for a minimum of 8 hours/day. B: an example of an Athome personalized workstation.

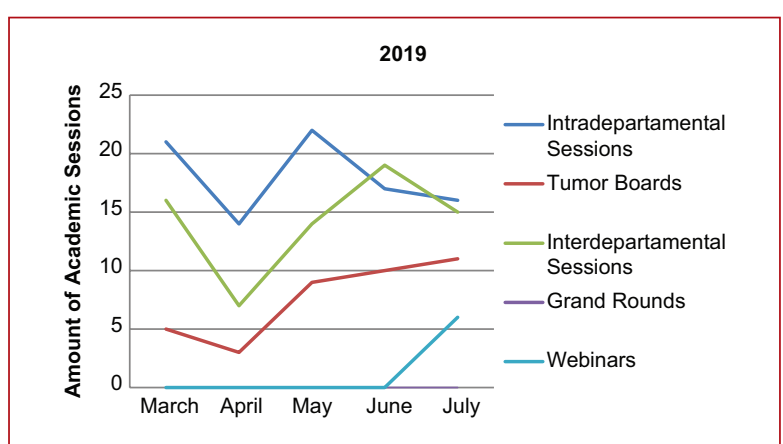

Figure 2. Departmental academic activities from March to July 2019.

For May 2020, the department nearly performed the same number of academic sessions as in May 2019 (44 vs. 45). By June 2020, total activities underwent an increase of $65.2 \%$ compared to June 2019 (76 vs. 46). Gains were further amplified in July 2020 , in which the total number of academic activities more than doubled compared to July 2019 (101 vs. 48).

\section{Discussion}

RDs should rapidly deploy technology solutions that allow radiologists to contribute remotely at distant work sites or from home, including examination protocolling, interpretation, and multidisciplinary case discussions. For

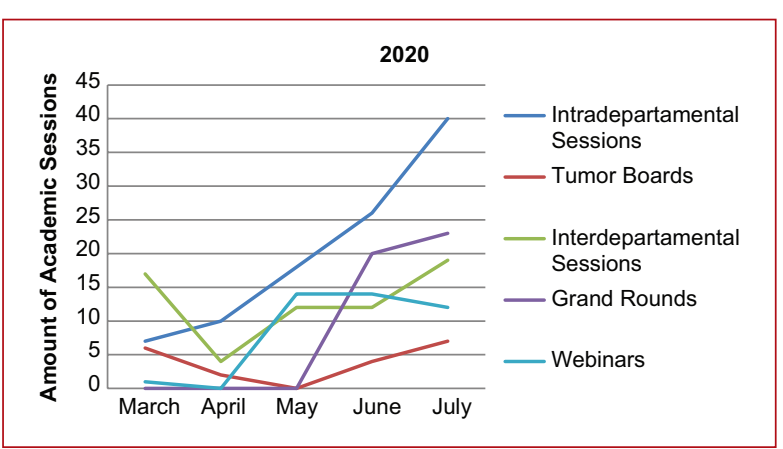

Figure 3. Departmental academic activities from March to July 2020.

trainees, these measures may mean a decreased need for on-site work and increased scheduling flexibility ${ }^{1}$.

\section{Remote learning}

Literature has well elucidated the efficacy of remote learning protocols, but information regarding its use at the level of a radiology residency is deficient ${ }^{2}$. Furthermore, remotely instituted workstations represent a novel approach to resident education. In this way, residents can continue diagnostic interpretation of various imaging modalities, including $\mathrm{X}$-ray, magnetic resonance imaging, and computed tomography (CT). Remotely accessible films preserve patient care continuity, this being especially 
important for patients requiring serial imaging or those with complex pathologies. Following hospital exposure, a 2-week period of home-based learning is paramount and coincides with nationally recommended quarantine guidelines. Should COVID-19 be contracted during hospital rounds, symptoms are likely to present within the 2-week home period before the resident would typically return.

Microsoft Teams allows for real-time interpretation of radiological studies and crucial interactions between trainees and more experienced colleagues ${ }^{3}$. Furthermore, YouTube Live permits the continuance of daily morning sessions while adhering to social distancing recommendations. The ease with which the department implemented such software may represent a favorable indication of their various utilities.

The department has also become significantly more active in other regards, including increasing participation in virtually held national and international seminars. This intervention is of particular importance for trainees, as it helps to facilitate continued educational discourse between otherwise isolated colleagues. This intervention may have extended value, as it mitigates many challenges associated with physical attendance of such events, such as travel and lodging costs.

\section{Competence evaluation}

Before COVID-19, residents were evaluated by senior physicians using written forms requiring in-person attendance. Since the pandemic, senior physicians have conducted evaluations in a virtual format utilizing the previously mentioned resident workstations. Residents are provided feedback by two senior physicians on concluding radiological assessments, which test a trainee's ability to interpret and describe image findings accurately. Interestingly, this adaptation offers a potential benefit compared to written evaluations. Feedback is more rapid and trainees, regardless of location, can follow the identification of crucial pathologies by more experienced radiologists in real time ${ }^{4}$. After the resolution of the pandemic, studies should explore the efficacy of implementing this feedback modality.

Given that the total number of images interpreted by trainees has decreased due to COVID-19, it is essential to consider potential resultant consequences. The "Core Exam" and "Certifying Exam" are nationally recognized measures of diagnostic radiology competence. The certifying college administers the tests after 36 months of residency training and 15 months after completion of residency, respectively. A correlation between the number of images interpreted during training and subsequent test score has been established ${ }^{5}$, but the influence of other modalities' compensatory use as described in this manuscript is unknown. Future investigations to compare scores achieved by pre- and post-pandemic cohorts are theoretically possible and may reveal any COVID-induced deficiencies, should they be present.

\section{Academic activities}

At the start of the pandemic, academic activities were significantly affected (Fig. 3). The RD experienced decreases in total activities for March and April versus 2019 (Fig. 2). Such declines were transient, however, and would ultimately recover in May. By June and July, the gross number of academic activities would far exceed levels seen in 2019. The growth sustained in this specific activity was theatrical. This growth may be an early indication that the RD maintains certain changes in operations more easily or that the RD believes Grand Rounds to play a more significant role in departmental functionality, so the RD emphasized them it is also important to note the asymmetric increase in some activities' frequency over others, Grand Rounds being an example.

Mandatory morning sessions were broadcast virtually by Microsoft Teams and YouTube Live, allowing homebased residents and faculty to attend and interact in real time. Similarly, the sessions were recorded and posted to the RD's YouTube channel, easily accessible to attendees and the public.

The RD held virtual Grand Rounds in place of traditional auditorium gatherings. This measure facilitated discussion by residents on critical radiological cases managed by the department. The use of a virtual format has been well-received by residents. According to a recently administered survey, $97 \%$ of residents indicated that they were "satisfied" or "very satisfied" with Grand Rounds, despite the format change ${ }^{3}$.

The use of a "healthy hour" by residents and radiologists has been strongly encouraged amidst the pandemic to promote mental well-being and minimize elevated stress due to the pandemic. Encouraged activities included exercising, yoga, literary reading, and meditation.

\section{Conclusion}

Radiologist participation in patient healthcare is an important determinant of patient outcome. Hospital presence, while currently reduced, should not hinder rendering essential radiology services. As such, adaptations undertaken to mitigate viral spread must be efficacious and cause minimal changes in clinical output. 
Resident training can and must continue during the pandemic. Remote technologies allow for acquisition of medical knowledge and evaluation of trainee progression while significantly reducing viral transmission. Adaptations such as telemedicine, teleradiology, and virtual interdepartmental interactions have now become necessary but may persist as standard inclusions in the deliverance of radiologic services.

\section{Limitations}

It is important to note that changes undertaken by the Autonomous University of Nuevo León's RD may not be applicable in every circumstance. Those with restricted remote internet access and those without the necessary hardware and software may find difficulty implementing such changes ${ }^{6}$. Furthermore, the university is a major academic institution. Programs with reduced staff and trainee populations may be unable to sustain implemented modifications to the degree described in this article. Despite these limitations, the principles, and their derived changes, serve as valuable lessons for all attempting to mount an effective pandemic response.

\section{Conflicts of interest}

The authors declare no conflicts of interest.

\section{Funding}

No financial sources were required.

\section{Ethical disclosures}

Protection of human and animal subjects. The authors declare that no experiments were performed on humans or animals for this study.

Confidentiality of data. The authors declare that no patient data appears in this article.

Right to privacy and informed consent. The authors declare that no patient data appears in this article.

\section{References}

1. Alvin MD, George E, Deng F, Warhadpande S, Lee SI. The impact of COVID-19 on radiology trainees. Radiology. 2020;296:246-8.

2. Awan OA, Shaikh F, Kalbfleisch B, Siegel EL, Chang P. RSNA diagnosis live: a novel web-based audience response tool to promote evidence-based learning. Radiographics. 2017;37:1111-8.

3. Chan HK, Lee, AL. Teaming up for patient care seminars: using Microsoft Teams private channels to facilitate HIPAA-compliant small group educational sessions. J Dent Educ. 2020;2020:1-3

4. Choi HH, Clark J, Jay AK, Filice RW. Minimizing barriers in learning for on-call radiology residents end-to-end web-based resident feedback system. J Digit Imaging. 2018;31:117-23.

5. Nickerson JP, Koski C, Anderson JC, Beckett B, Jackson VP. Correlation between radiology ACGME case logs values and ABR core exam pass rate. Acad Radiol. 2020;27:269-73.

6. Maru DS, Schwarz R, Basu SA, Sharma A, Moore C. Turning a blind eye: the mobilization of radiology services in resource-poor regions. Glob Health. 2010;6:18 